Research Article

\title{
Approximate Analytic Solutions of Two-Dimensional Nonlinear Klein-Gordon Equation by Using the Reduced Differential Transform Method
}

\author{
Wayinhareg Gashaw Belayeh $\left(\mathbb{D}\right.$, Yesuf Obsie Mussa ${ }^{(D}$, and Ademe Kebede Gizaw \\ Department of Mathematics, College of Natural Sciences, Jimma University, Jimma, Ethiopia \\ Correspondence should be addressed to Ademe Kebede Gizaw; kebedeademe2020@gmail.com
}

Received 20 August 2020; Revised 17 November 2020; Accepted 26 November 2020; Published 15 December 2020

Academic Editor: Nadeem Ahmad Sheikh

Copyright (c) 2020 Wayinhareg Gashaw Belayeh et al. This is an open access article distributed under the Creative Commons Attribution License, which permits unrestricted use, distribution, and reproduction in any medium, provided the original work is properly cited.

\begin{abstract}
In this paper, the reduced differential transform method (RDTM) is successfully implemented for solving two-dimensional nonlinear Klein-Gordon equations (NLKGEs) with quadratic and cubic nonlinearities subject to appropriate initial conditions. The proposed technique has the advantage of producing an analytical approximation in a convergent power series form with a reduced number of calculable terms. Two test examples from mathematical physics are discussed to illustrate the validity and efficiency of the method. In addition, numerical solutions of the test examples are presented graphically to show the reliability and accuracy of the method. Also, the results indicate that the introduced method is promising for solving other type systems of NLPDEs.
\end{abstract}

\section{Introduction}

In many applications of science and engineering such as fluid dynamics, plasma physics, hydrodynamics, solid-state physics, optical fibers, acoustics, and other disciplines, the nonlinear equations appear for modeling physical phenomenon [1]. Recently, a lot of research was carried out on real-life applications modeled by PDEs [2-12]. The Klein-Gordon equation is an important class of partial differential equations and arises in relativistic quantum mechanics and field theory, which is of great importance for the high-energy physicists [13], and is used to model many different phenomena, including the propagation of dislocations in crystals and the behavior of elementary particles [14].

The $\mathrm{n}$-dimensional nonlinear Klein-Gordon equation is given by partial differential equation [15]:

$$
\begin{aligned}
u_{t t}(\mathbf{x}, t)+\beta u_{t}(\mathbf{x}, t)= & \alpha\left(u_{x x}(\mathbf{x}, t)+u_{y y}(\mathbf{x}, t)\right) \\
& -g(u(\mathbf{x}, t))+f(\mathbf{x}, t), \quad \mathbf{x} \in \Omega, t>0,
\end{aligned}
$$

$$
\begin{aligned}
u(\mathbf{x}, 0) & =\varphi_{1}(\mathbf{x}), \\
u_{t}(\mathbf{x}, 0) & =\varphi_{2}(\mathbf{x}), \quad \mathbf{x} \in \Omega,
\end{aligned}
$$

where $\Omega$ is a domain of $R^{n}(n=1,2, \ldots), \quad \mathbf{x}=\left(x_{1}\right.$, $\left.x_{2}, \ldots, x_{n}\right)^{T}$ is the space variable, $t$ is the time variable, $u(\mathbf{x}, t)$ is the unknown function, $g(u)$ is a nonlinear function of $u$ with different types of nonlinearities, $f(\mathbf{x}, t)$ is a given function, $\varphi_{1}(\mathbf{x})$ and $\varphi_{2}(\mathbf{x})$ are prescribed initial functions, and $\alpha$ and $\beta$ are known constants.

In the present work, we are dealing with the approximate analytical solution of the nonlinear Klein-Gordon equation (1) with a nonlinear function $g(u)$ having the form $g(u)=$ $\gamma_{1} u+\gamma_{2} u^{m}$.

That is,

$$
\begin{aligned}
\frac{\partial^{2} u(x, y, t)}{\partial t^{2}}+\beta \frac{\partial u(x, y, t)}{\partial t}= & \alpha\left(\frac{\partial^{2} u(x, y, t)}{\partial x^{2}}+\frac{\partial^{2} u(x, y, t)}{\partial y^{2}}\right) \\
& -\left(\gamma_{1} u(x, y, t)+\gamma_{2} u^{m}(x, y, t)\right) \\
& +f(x, y, t),
\end{aligned}
$$

with initial conditions 
subject to the initial conditions

$$
\begin{aligned}
u(x, y, 0) & =g_{1}(x, y), \\
u_{t}(x, y, 0) & =g_{2}(x, y),
\end{aligned}
$$

where $t>0$ and $(x, y) \in R^{2}$.

Remark 1. Equation (3) is called the Klein-Gordon equation with quadratic nonlinearity if $m=2$ and with cubic nonlinearity if $m=3$.

In recent years, many researchers have paid attention to study the solutions of NLPDEs by various methods (see [16] and the references therein).

The reduced differential transform method (RDTM) was first proposed by Keskin and Oturanc [17] for solving partial differential equations (PDEs). It is an iterative procedure based on the use of the Taylor series solution of differential equations. RDTM has been successfully applied to solve various nonlinear partial differential equations [18-28].

The main aim of this study is to obtain the approximate analytical solutions for two-dimensional nonlinear Klein-Gordon equation (NLKGE) with quadratic and cubic nonlinearities, since most of the research focused on the numerical solutions for this problem. The reduced differential transform method is used for this purpose for several reasons. The first reason is that the method has not previously been applied to solve this problem. Secondly, this method can directly be applied to NLKGE. Thirdly, this method can reduce the size of the calculations and can provide an analytic approximation, in many cases exact solutions, in rapidly convergent power series form with elegantly computed terms (see [29] and the references therein). Moreover, the reduced differential transform method (RDTM) has an alternative approach of solving problems to overcome the demerit of discretization, linearization, or perturbations of well-known numerical and analytical methods such as Adomian decomposition, differential transform, homotopy perturbation, and variational iteration $[29,30]$.

The structure of the remaining parts of this paper is organized as follows. In Section 2, we begin with some basic definitions and describe the proposed method. In Section 3, we discuss the convergence analysis of the method. In Section 4, we apply the reduced differential transform method to solve two illustrative examples in order to show its ability and efficiency. Concluding remarks are given in Section 5.

\section{Reduced Differential Transform Method}

The basic definitions and operations of the two-dimensional reduced differential transform method [30-34] are introduced as follows.

Definition 1. If the function $u(x, y, t)$ is analytic and differentiable continuously with respect to time $t$ and space in the domain of interest, then

$$
U_{k}(x, y)=\frac{1}{k !}\left[\frac{\partial^{k} u(x, y, t)}{\partial t^{k}}\right]_{t=t_{0}},
$$

where the $t$-dimensional spectrum function $U_{k}(x, y)$ is the transformed function of $u(x, y, t)$.

In this study, the lower case $u(x, y, t)$ represents the original function while the upper case $U_{k}(x, y)$ stands for the transformed function.

Definition 2. The inverse differential transform of $U_{k}(x, y)$ is defined as

$$
u(x, y, t)=\sum_{k=0}^{\infty} U_{k}(x, y)\left(t-t_{0}\right)^{k} .
$$

Combining equations (5) and (6), we obtain

$$
u(x, y, t)=\sum_{k=0}^{\infty} \frac{1}{k !}\left[\frac{\partial^{k} u(x, y, t)}{\partial t^{k}}\right]_{t=t_{0}} t^{k}
$$

Note that the function $u(x, y, t)$ can be written in a finite series as follows:

$$
u_{n}(x, y, t)=\sum_{k=0}^{n} U_{k}(x, y)\left(t-t_{0}\right)^{k}+R_{n}(x, y, t),
$$

where the tail function $R_{n}(x, y, t)$ is negligibly small. Therefore, the exact solution of the problem is given by

$$
u(x, y, t)=\lim _{n \longrightarrow \infty} u_{n}(x, y, t) .
$$

Lemma 1. If $w(x, y, t)=u^{2}(x, y, t)$, then $W_{k}(x, y)=$ $\sum_{i=0}^{k} U_{i}(x, y) U_{k-i}(x, y)$.

Proof. By using Table 1, we have

$$
\begin{aligned}
W_{k}(x, y)= & \frac{1}{k !}\left[\frac{\partial^{k}(u(x, y, t) u(x, y, t))}{\partial t^{k}}\right]_{t=0} \\
= & \frac{1}{k !}\left[u(x, y, t) \frac{\partial^{k} u(x, y, t)}{\partial t^{k}}+k \frac{\partial u(x, y, t)}{\partial t} \frac{\partial^{k-1} u(x, y, t)}{\partial t^{k-1}}+\frac{k(k-1)}{2 !} \frac{\partial^{2} u(x, y, t)}{\partial t^{2}} \frac{\partial^{k-2} u(x, y, t)}{\partial t^{k-2}}\right. \\
& \left.+\ldots+k \frac{\partial^{k-1} u(x, y, t)}{\partial t^{k-1}} \frac{\partial u(x, y, t)}{\partial t}+\frac{\partial^{k} u(x, y, t)}{\partial t^{k}} u(x, y, t)\right]_{t=0}
\end{aligned}
$$


TABLE 1: Basic operations of RDTM.

\begin{tabular}{lc}
\hline Original function & Transformed function \\
\hline$u(x, y, t)$ & $\left.U_{k}(x, y)=\frac{1}{k !} \partial^{k} u(x, y, t) / \partial t^{k}\right]_{t=0}$ \\
$w(x, y, t)=\alpha u(x, y, t)+\beta v(x, y, t)$ & $W_{k}(x, y)=\alpha U_{k}(x, y)+\beta V_{k}(x, y)$, \\
& where $\alpha$ and $\beta$ are constants. \\
$w(x, y, t)=u(x, y, t) v(x, y, t)$ & $W_{k}(x, y)=\sum_{r=0}^{\infty} U_{r}(x, y) V_{k-r}(x, y)$ \\
& $=\sum_{r=0}^{\infty} V_{k-r}(x, y) U_{r}(x, y)$ \\
$w(x, y, t)=\partial^{r} / \partial t^{r} u(x, y, t)$ & $W_{k}(x, y)=(k+r) ! / k ! U_{k+r}(x, y)$ \\
$w(x, y, t)=\partial^{2} / \partial x^{2} u(x, y, t)$ & $W_{k}(x, y)=\partial^{2} / \partial x^{2} U_{k}(x, y)$ \\
$w(x, y, t)=\partial^{2} / \partial y^{2} u(x, y, t)$ & $W_{k}(x, y)=\partial^{2} / \partial y^{2} U_{k}(x, y)$ \\
$w(x, y, t)=\sin (\alpha x+\gamma y+\beta t)$ & \\
$w(x, y, t)=\cos (\alpha x+\gamma y+\beta t)$ & $W_{k}(x, y)=\beta^{k} / k ! \sin (\alpha x+\gamma y+k \pi / 2)$, where $\alpha, \beta$ and $\gamma$ are constants. \\
\hline
\end{tabular}

By Leibnitz's theorem, this can be written as

$$
\begin{aligned}
W_{k}(x, y)= & {\left[\left(\frac{1}{k !} \frac{\partial^{0} u(x, y, t)}{\partial t^{0}} \frac{\partial^{k} u(x, y, t)}{\partial t^{k}}\right) \frac{1}{0 !}+\left(\frac{1}{(k-1) !} \frac{\partial u(x, y, t)}{\partial t} \frac{\partial^{k-1} u(x, y, t)}{\partial t^{k-1}}\right) \frac{1}{1 !}\right.} \\
& +\left(\frac{1}{(k-2) !} \frac{\partial u(x, y, t)}{\partial t} \frac{\partial^{k-2} u(x, y, t)}{\partial t^{k-2}}\right) \frac{1}{2 !}+\cdots+\left(\frac{1}{(k-1) !} \frac{\partial^{k-1} u(x, y, t)}{\partial t^{k-1}} \frac{\partial u(x, y, t)}{\partial t}\right) \frac{1}{1 !} \\
& \left.+\left(\frac{1}{k !} \frac{\partial^{k} u(x, y, t)}{\partial t^{k}} \frac{\partial^{0} u(x, y, t)}{\partial t^{0}}\right) \frac{1}{0 !}\right]_{t=0} \\
= & \sum_{i=0}^{k} \frac{1}{i !(k-i) !}\left[\frac{\partial^{i} u(x, y, t)}{\partial t^{i}} \frac{\partial^{k-i} u(x, y, t)}{\partial t^{k-i}}\right]_{t=0} \\
= & \sum_{i=0}^{k} \frac{1}{i !}\left[\frac{\partial^{i} u(x, y, t)}{\partial t^{i}}\right]_{t=0} \frac{1}{(k-i) !}\left[\frac{\partial^{k-i} u(x, y, t)}{\partial t^{k-i}}\right]_{t=0} \\
= & \sum_{i=0}^{k} U_{i}(x, y) U_{k-i}(x, y) .
\end{aligned}
$$

Lemma 2. If $f(x, y, t)=u^{3}(x, y, t)$, then $W_{k}(x, y)=$ $\sum_{j=0}^{k} \sum_{i=0}^{j} U_{i}(x, y) U_{j-i}(x, y) U_{k-j}(x, y)$.

Proof. Observe that $u^{3}(x, y, t)=u^{2}(x, y, t) u(x, y, t)$. So, by using Table 1, we have

$$
\begin{aligned}
W_{k}(x, y) & =\frac{1}{k !}\left[\frac{\partial^{k}}{\partial t^{k}}\left(u^{3}(x, y, t)\right)\right]_{t=0} \\
& =\frac{1}{k !}\left[\frac{\partial^{k}}{\partial t^{k}} u^{2}(x, y, t) u(x, y, t)\right]_{t=0} \\
& =\sum_{j=0}^{k} F_{j}(x, y) U_{k-j}(x, y),
\end{aligned}
$$

where $F_{j}$ is the reduced differential transform of $u^{2}(x, y, t)=\sum_{i=0}^{j} U_{i}(x, y) U_{j-i}(x, y)$ (Lemma 1$)$.

Thus,

$$
\begin{aligned}
W_{k}(x, y) & =\sum_{j=0}^{k} F_{j}(x, y) U_{k-j}(x, y) \\
& =\sum_{j=0}^{k} \sum_{i=0}^{j} U_{i}(x, y) U_{j-i}(x, y) U_{k-j}(x, y) .
\end{aligned}
$$

To demonstrate the basic concepts of RDTM, we consider the two-dimensional nonlinear Klein-Gordon equation from equation (3) subject to the initial conditions given by equation (4).

By RDTM, from equation (3), we obtain the following recurrence relation:

$$
\begin{aligned}
U_{k+2}(x, y)= & \frac{1}{(k+1)(k+2)}\left[\alpha\left(\frac{\partial^{2} U_{k}(x, y)}{\partial x^{2}}+\frac{\partial^{2} U_{k}(x, y)}{\partial y^{2}}\right)\right. \\
& \left.-\beta U_{k}(x, y)-N_{k}(x, y)+F_{k}(x, y)\right],
\end{aligned}
$$


where $N_{k}(x, y)$ and $F_{k}(x, y)$ are the transformed forms of the nonlinear terms $g(u)$ and $f(x, y, t)$, respectively. Similarly, by RDTM, from the initial condition (4), we get

$$
\begin{aligned}
& U_{0}(x, y)=g_{1}(x, y), \\
& U_{1}(x, y)=g_{2}(x, y),
\end{aligned}
$$

respectively.

Substituting equation (15) into equation (14), we get the set of values $\left\{U_{k}(x, y)\right\}_{k=0}^{n}$. Then, the inverse reduced differential transform of these sets of values provides the n-term approximate solution as

$$
\tilde{u}_{n}(x, y, t)=\sum_{k=0}^{n} U_{k}(x, t) t^{k} .
$$

Therefore, the exact solution of the problem is given by

$$
u(x, y, t)=\lim _{n \longrightarrow \infty} \widetilde{u}_{n}(x, y, t) .
$$

\section{Convergence Analysis}

In this section, the convergence analysis of the approximate analytical solutions which are computed from the application of RDTM [30] is presented.

Let us consider equation (3) in the following functional equation form:

$$
u(x, y, t)=F(u(x, y, t)),
$$

where $F$ is a general nonlinear operator involving both linear and nonlinear terms.

According to RDTM, equation (3) has a solution of the form

$$
u(x, y, t)=\sum_{k=0}^{\infty} U_{k}(x, y) t^{k}=\sum_{k=0}^{\infty} \beta_{k} .
$$

It is noted that the solutions by RDTM are equivalent to determining the sequences

$$
\begin{aligned}
S_{0}= & U_{0}(x, y)=\beta_{0}, \\
S_{1}= & U_{0}(x, y)+U_{1}(x, y) t=\beta_{0}+\beta_{1}, \\
S_{2}= & U_{0}(x, y)+U_{1}(x, y) t+U_{2}(x, y) t^{2} \\
= & \beta_{0}+\beta_{1}+\beta_{2}, \\
& \cdots \\
S_{n}= & \sum_{k=0}^{n} U_{k}(x, y) t^{k}=\sum_{k=0}^{n} \beta_{k},
\end{aligned}
$$

by using iterative scheme

$$
S_{n+1}=F\left(S_{n}\right),
$$

associated with the functional equation

$$
S=F(S) \text {. }
$$

Hence, the solution obtained by RDTM, $u(x, y, t)=$ $\sum_{k=0}^{\infty} U_{k}(x, y) t^{k}=\sum_{k=0}^{\infty} \beta_{k}$, is equivalent to $\left\{S_{n}\right\}_{n=0}^{\infty}$.
The sufficient condition for convergence of the series solution $\left\{S_{n}\right\}_{n=0}^{\infty}$ is given in the following theorem.

Theorem 1. Let $F$ be an operator from a from Hilbert space $H$ into $H$. Then, the series solution $\left\{S_{n}\right\}_{n=0}^{\infty}$ converges whenever there is " $\alpha$ " such that $0<\alpha<1$, and $\left\|\beta_{k+1}\right\| \leq \alpha\left\|\beta_{k}\right\|$ (see for proof Ref. [30]).

Theorem 2. Let $F$ be a nonlinear operator satisfying Lipschitz condition from Hilbert space $H$ into $H$ and $u(x, y, t)$ be exact solution of the given NLKGE. If the series solution $\left\{S_{n}\right\}_{n=0}^{\infty}$ converges, then it converges to $u(x, y, t)$ (see for proof Ref. [30]).

Definition 3. For $k \in N \bigcup\{0\}$, we define

$$
\alpha_{k}= \begin{cases}\frac{\left\|\beta_{k+1}\right\|}{\left\|\beta_{k}\right\|}=\frac{\left\|U_{k+1}(x, y) t^{k+1}\right\|}{\left\|U_{k}(x, y) t^{k}\right\|}, & \text { if }\left\|\beta_{k}\right\|=\left\|U_{k}(x, y) t^{k}\right\| \neq 0, \\ 0, & \text { if }\left\|\beta_{k}\right\|=\left\|U_{k}(x, y) t^{k}\right\|=0 .\end{cases}
$$

Then, we can say that the series approximate solution $\left\{S_{n}\right\}_{n=0}^{\infty}$ converges to the exact solution $u(x, y, t)$ when $0 \leq \alpha_{k}<1$ for $k=0,1,2, \ldots$

\section{Test Examples}

In this section, we consider two test examples that demonstrate the performance and efficiency of the RDTM for solving two-dimensional inhomogeneous nonlinear Klein-Gordon equations.

Example 1. Consider the two-dimensional NLKGE (3) with $m=2, \alpha=1, \beta=0, \gamma_{1}=0, \gamma_{2}=1$.

That is,

$$
\begin{aligned}
& u_{t t}(x, y, t)-u_{x x}(x, y, t)-u_{y y}(x, y, t)+u^{2}(x, y, t) \\
& =-x y \cos t+x^{2} y^{2} \cos ^{2} t, \quad t>0,
\end{aligned}
$$

with initial conditions

$$
\begin{aligned}
u(x, y, 0) & =x y, \\
u_{t}(x, y, 0) & =0 .
\end{aligned}
$$

Applying properties of RDTM to equation (24), we obtain the following recurrence relation:

$$
U_{k+2}(x, y)=\frac{1}{(k+1)(k+2)}\left[\frac{\partial^{2} U_{k}(x, y)}{\partial x^{2}}+\frac{\partial^{2} U_{k}(x, y)}{\partial y^{2}}\right.
$$

$$
\left.-N_{k}(x, y)+F_{k}(x, y)\right]
$$

where

$$
N_{k}(x, y)=\sum_{r=0}^{k} U_{r}(x, y) U_{k-r}(x, y)
$$


is the transformed form of $u^{2}(x, y, t)$ and $F_{k}(x, y)$ is the transformed form of the function $f(x, y, t)=$ $-x y \cos t+x^{2} y^{2} \cos ^{2} t$.

By similar scheme from (25), we obtain

$$
\begin{aligned}
& U_{0}(x, y)=x y, \\
& U_{1}(x, y)=0 .
\end{aligned}
$$

Let

$$
g(x, y, t)=-x y \cos t
$$

and

$$
h(x, y, t)=g^{2}(x, y, t)=x^{2} y^{2} \cos ^{2} t .
$$

Applying properties of RDTM on equations (29) and (30), we get

$$
G_{k}(x, y)=-x y \frac{1}{k !} \cos \left(k \frac{\pi}{2}\right)
$$

and

$$
H_{k}(x, y)=\sum_{i=0}^{k} G_{i}(x, y) G_{k-i}(x, y)
$$

respectively.

Thus,

$$
F_{k}(x, y)=G_{k}(x, y)+H_{k}(x, y) .
$$

We then compute the set of values of $\left\{u_{k}(x, y)\right\}_{k=0}^{n}$.

Substituting equations (27), (31), and (32) into equation (26) with $k=0,1,2, \cdots, n$, we obtain

$$
\begin{aligned}
& N_{0}(x, y)=\sum_{r=0}^{0} U_{r} U_{k-r}=U_{0}^{2}(x, y)=x^{2} y^{2}, \\
& G_{0}(x, y)=-x y, \\
& H_{0}(x, y)=\sum_{i=0}^{0} G_{i}(x, y) G_{k-i}(x, y)=G_{0}^{2}(x, y)=x^{2} y^{2},
\end{aligned}
$$

respectively.

So,

$$
F_{0}(x, y)=G_{0}(x, y)+H_{0}(x, y)=-x y+x^{2} y^{2} .
$$

Hence, from equation (26), we obtain

$$
U_{2}(x, y)=\frac{1}{2}\left[0+0-x^{2} y^{2}+x^{2} y^{2}-x y\right]=-\frac{x y}{2} .
$$

Accordingly,

$$
\begin{aligned}
& N_{1}(x, y)=2 U_{1}(x, y) U_{0}(x, y)=0, \\
& G_{1}(x, y)=0 \\
& H_{1}(x, y)=\sum_{i=0}^{1} G_{i}(x, y) G_{k-i}(x, y)=0 .
\end{aligned}
$$

Thus, $F_{1}(x, y)=G_{1}(x, y)+H_{1}(x, y)=0$, and hence when $k=1$, equation (26) yields
$U_{3}(x, y)=\frac{1}{3 !}\left[\frac{\partial^{2} U_{1}(x, y)}{\partial x^{2}}+\frac{\partial^{2} U_{1}(x, y)}{\partial y^{2}}-N_{1}(x, y)+F_{1}(x, y)\right]=0$

Using similar procedure, we obtain the following results:

$$
\begin{aligned}
& U_{4}(x, y)=\frac{x y}{4 !}, \\
& U_{5}(x, y)=0, \\
& U_{6}(x, y)=-\frac{x y}{6 !},
\end{aligned}
$$

and so on.

Substituting all these values in equation (16), we obtain

$$
\begin{aligned}
u(x, y, t) & =\sum_{k=0}^{n} U_{k}(x, y) t^{k} \\
& =x y\left(1-\frac{1}{2 !} t^{2}+\frac{1}{4 !} t^{4}-\frac{1}{6 !} t^{6}+\cdots+\frac{(-1)^{n}}{(2 n) !} t^{(2 n)}\right),
\end{aligned}
$$

and hence by equation (17), the exact solution of Example 1 is $u(x, y, t)=x y \cos t$.

To make a decision on the convergence of the solution, we compute $\alpha_{k}$ using Definition 3 and Theorem 1 as follows.

First, let us take $x=y=t=1$; then,

$$
\begin{aligned}
& \alpha_{0}=\frac{\beta_{1}}{\beta_{0}}=\frac{U_{1}(x, y) t^{1}}{U_{0}(x, y) t^{0}}=\frac{0 \times 1^{1}}{1 \times 1}=0<1 \\
& \alpha_{1}=\frac{\beta_{2}}{\beta_{1}}=0<1, \text { since }\left\|\beta_{1}\right\|=0, \text { definition 3.1, } \\
& \alpha_{2}=\frac{\beta_{3}}{\beta_{2}}=\frac{U_{3}(x, y) t^{3}}{U_{2}(x, y) t^{2}}=\frac{0 \times 1^{3}}{1 / 2 ! \times 1 \times 1 \times 1^{2}}=0<1, \\
& \alpha_{3}=\frac{\beta_{4}}{\beta_{3}}=0<1, \text { since } \beta_{3}=U_{3}(x, y) t^{3}=0,
\end{aligned}
$$

Hence $x, y, t \geq 0, \alpha_{0}=\alpha_{1}=\alpha_{2}=\alpha_{3}=\ldots \alpha_{\infty}=0<1$.

Therefore, by Theorem 2, the solution of the given NLKGE by RDTM has a convergent solution.

Figures representing the numerical solution of Example 1 are presented below.

Figures 1(a) and 1(b), respectively, show the physical behavior of the approximate solution by RDTM of Example 1 at $t=0.1$ for equal values of $x$ and $y$ in $[0,3.2]$ and the associated absolute errors. From these figures, it can be observed that the numerical results by RDTM are in excellent agreement with the exact solution of the problem under consideration.

In Figure 1(c), we have plotted absolute errors by the proposed method corresponding to $t=5.0,5.2,5.4,5.6$ and $t=5.8$. This figure represents the errors of numerical results by RDTM that could appear for the long time range. 


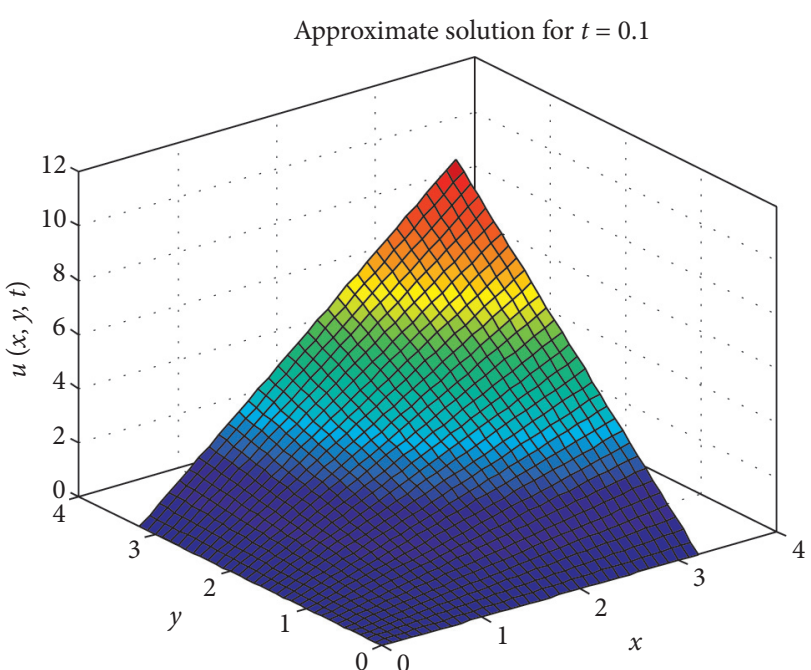

(a)

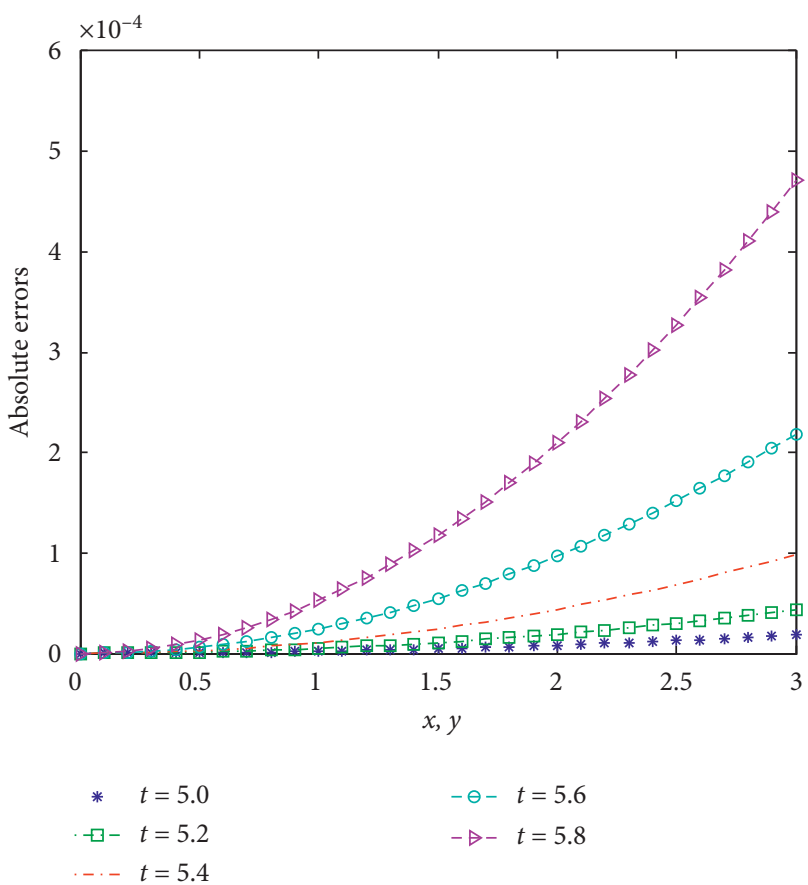

(c)

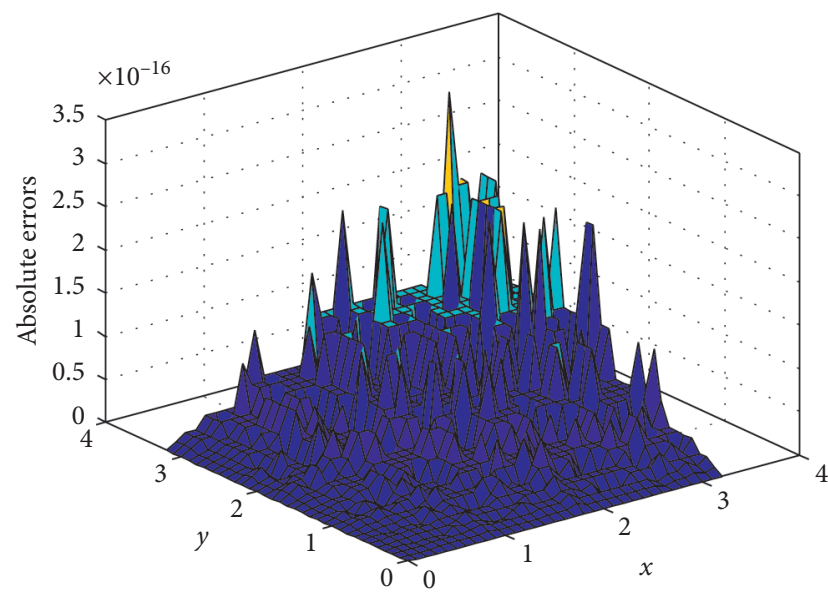

(b)

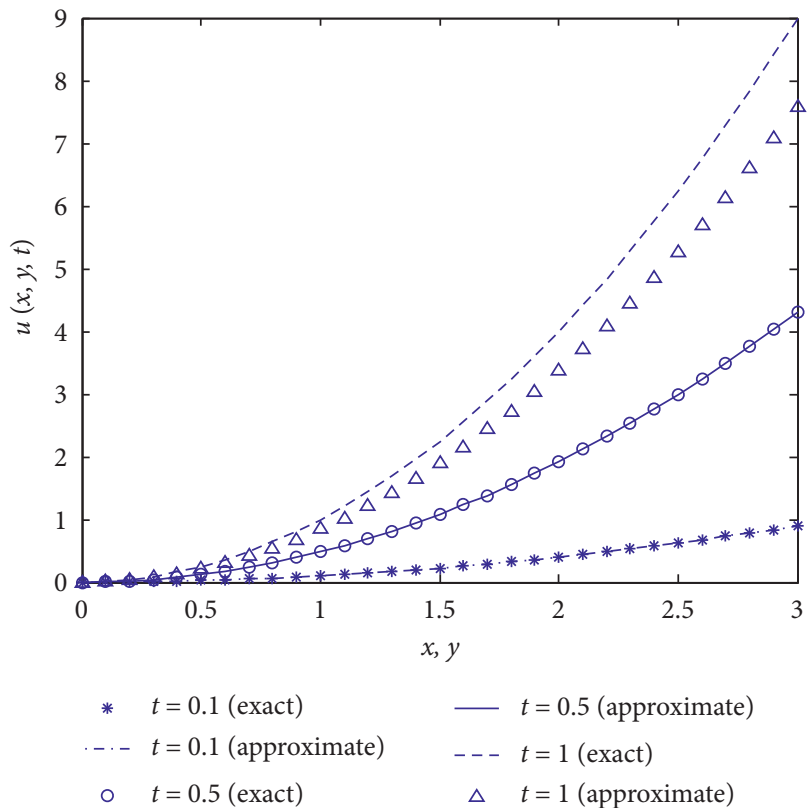

(d)

Figure 1: Plots of the solution behavior of Example 1. (a) Approximate solution at $t=0.1$. (b) Absolute errors at $t=0.1$. (c) Absolute errors for different values of $t$. (d) Comparison of exact and approximate solutions for different values of $t$.

In Figure 1(d), we have compared the approximate solutions by RDTM and exact solutions corresponding to $t=0.1,0.5$ and $t=1$, at equal values of $x$ and $y$ in [0,3.2]. This figure asserts that the numerical results obtained by RDTM are in a good agreement with the exact results, especially for $t<0.5$. In general, Figures 1 (a) -1 (d) show convergence of the approximate solution obtained by RDTM to the exact solution rapidly.

Example 2. Consider the two-dimensional NLKGE (3) with $m=3, \alpha=1, \beta=0, \gamma_{1}=0, \gamma_{2}=1, \quad$ and $\quad f(x, y, t)=$ $\cos x \cos y \sin t+\cos ^{3} x \cos ^{3} y \sin ^{3} t$.
That is,

$$
\begin{gathered}
u_{t t}(x, y, t)-u_{x x}(x, y, t)-u_{y y}(x, y, t)+u^{3}(x, y, t) \\
=\cos x \cos y \sin t+\cos ^{3} x \cos ^{3} y \sin ^{3} t, \quad t>0,
\end{gathered}
$$

with initial conditions

$$
\begin{aligned}
u(x, y, 0) & =0, \\
u_{t}(x, y, 0) & =\cos x \cos y .
\end{aligned}
$$

By applying RDTM on equation (42), the following recursive equation is obtained: 


$$
\begin{aligned}
U_{k+2}(x, y)= & \frac{1}{(k+1)(k+2)}\left[\frac{\partial^{2} U_{k}(x, y)}{\partial x^{2}}+\frac{\partial^{2} U_{k}(x, y)}{\partial y^{2}}\right. \\
& \left.-N_{k}(x, y)+F_{k}(x, y)\right]
\end{aligned}
$$

where

$$
N_{k}(x, y)=\sum_{j=0}^{k} \sum_{i=0}^{j} U_{i}(x, y) U_{j-i}(x, y) U_{k-j}(x, y)
$$

is the transformed form of $u^{3}(x, y, t)$ and $F_{k}(x, y)$ is the transformed form of the function $f(x, y, t)=$ $\cos x \cos y \sin t+\cos ^{3} x \cos ^{3} y \sin ^{3} t$.

The reduced differential transform of equation (43) is

$$
\begin{aligned}
& U_{0}(x, y)=0, \\
& U_{1}(x, y)=\cos x \cos y,
\end{aligned}
$$

The transformed function $F_{k}$ can be well expressed as follows.

Let $g(x, y, t)=\cos x \cos y \sin t$

$$
\begin{aligned}
= & \frac{1}{4}[\sin (t+x-y)+\sin (t-x+y) \\
& +\sin (t+x+y)+\sin (t-x-y)],
\end{aligned}
$$

and

$$
h(x, y, t)=g^{3}(x, y, t)=\cos ^{3} x \cos ^{3} y \sin ^{3} t .
$$

Then, the reduced differential transform of the equations (47) and (48) is

$$
\begin{aligned}
G_{k}(x, y)= & \frac{1}{4}\left[\frac{1}{k !} \sin \left(k \frac{\pi}{2}+x-y\right)+\frac{1}{k !} \sin \left(k \frac{\pi}{2}-x+y\right)\right. \\
& \left.+\frac{1}{k !} \sin \left(k \frac{\pi}{2}+x+y\right)\right]+\frac{1}{4}\left[\frac{1}{k !} \sin \left(k \frac{\pi}{2}-x-y\right)\right],
\end{aligned}
$$

and

$$
H_{k}(x, y)=\sum_{j=0}^{k} \sum_{i=0}^{j} G_{i}(x, y) G_{j-i}(x, y) G_{k-j}(x, y),
$$

respectively.

Thus,

$$
F_{k}(x, y)=G_{k}(x, y)+H_{k}(x, y) .
$$

We now compute the set of values of $\left\{u_{k}(x, y)\right\}_{k=0}^{n}$.

When $k=0$, from equations (45), (49), and (50), we obtain

$$
\begin{aligned}
N_{0}(x, y)= & \sum_{j=0}^{0}\left(\sum_{i=0}^{j} U_{i} U_{j-i} U_{k-j}\right)=U_{0}^{3}(x, y)=0, \\
G_{0}(x, y)= & \frac{1}{4} \sin (x-y)+\sin (-x+y) \\
& +\sin (x+y)+\sin (-x-y)=0,
\end{aligned}
$$

$$
\begin{aligned}
H_{0}(x, y) & =\sum_{j=0}^{0} \sum_{i=0}^{0} G_{i}(x, y) G_{j-i}(x, y) G_{k-j}(x, y) \\
& =G_{0}^{3}(x, y)=0^{3}=0
\end{aligned}
$$

respectively.

Thus, $F_{0}(x, y)=G_{0}(x, y)+H_{0}(x, y)=0$, and therefore, equation (44) becomes

$$
U_{2}(x, y)=\frac{1}{2}\left[\frac{\partial^{2} U_{0}(x, y)}{\partial x^{2}}+\frac{\partial^{2} U_{0}(x, y)}{\partial y^{2}}-N_{0}(x, y)+F_{0}(x, y)\right]=\frac{1}{2}[0+0-0+0]=0
$$

When $k=1$, we have 


$$
\begin{aligned}
N_{1}(x, y) & =\sum_{j=0}^{1}\left(\sum_{i=0}^{j} U_{i}(x, y) U_{j-i}(x, y) U_{k-j}(x, y)\right)=3 U_{0}^{2}(x, y) U_{1}(x, y)=0, \\
\mathrm{G}_{1}(x, y) & =\frac{1}{8}\left[\sin \left(\frac{\pi}{2}+\mathrm{x}-\mathrm{y}\right)+\sin \left(\frac{\pi}{2}-(x-y)\right)+\sin \left(\frac{\pi}{2}+x+y\right)+\sin \left(\frac{\pi}{2}-(x+y)\right)\right], \\
& =\frac{1}{4}[2 \cos (x-y)+2 \cos (x+y)], \\
& =\cos x \cos y \\
H_{1}(x, y) & =\sum_{j=0}^{1} \sum_{i=0}^{j} G_{i}(x, y) G_{j-i}(x, y) G_{k-j}(x, y)=3 G_{0}^{2}(x, y) G_{1}(x, y)=0 .
\end{aligned}
$$

As a result, $F_{1}(x, y)=G_{1}(x, y)+H_{1}(x, y)=\cos x \cos y$. So, equation (44) implies

$$
\begin{aligned}
U_{3}(x, y) & =\frac{1}{6}\left[\frac{\partial^{2} U_{1}(x, y)}{\partial x^{2}}+\frac{\partial^{2} U_{1}(x, y)}{\partial y^{2}}-N_{1}(x, y)+F_{1}(x, y)\right] \\
& =\frac{1}{6}\left[\frac{\partial^{2} \cos x \cos y}{\partial x^{2}}+\frac{\partial^{2} \cos x \cos y}{\partial y^{2}}-0+\cos x \cos y\right] \\
& =-\frac{1}{3 !} \cos x \cos y .
\end{aligned}
$$

$$
U_{4}(x, y)=0
$$$$
U_{5}(x, y)=\frac{1}{5 !} \cos x \cos y
$$$$
U_{6}(x, y)=0 \text {, }
$$$$
U_{7}(x, y)=\frac{-1}{7 !} \cos x \cos y,
$$

and so on.

Then, the differential inverse transform of the set of values $\left\{U_{k}(x, y)\right\}_{k=0}^{n}$ is

In a similar manner, we obtain

$$
\begin{aligned}
u(x, y, t)= & \sum_{k=0}^{n} U_{k}(x, y) t^{k} \\
= & \cos x \cos y t^{1}-\frac{1}{3 !} \cos x \cos y t^{3}+\frac{1}{5 !} \cos x \cos y t^{5}-\frac{1}{7 !} \cos x \cos y t^{7}+\ldots+ \\
& +\frac{(-1)^{n}}{(2 n+1) !} \cos x \cos y t^{n} \\
= & \cos x \cos y\left(t^{1}-\frac{1}{3 !} t^{3}+\frac{1}{5 !} t^{5}-\frac{1}{7 !} t^{7}+\cdots+\frac{(-1)^{n}}{(2 n+1) !} t^{(2 n+1)}\right) .
\end{aligned}
$$

Therefore, by equation (17), the solution of Example 2 is $u(x, y, t)=\cos x \cos y \sin t$ which is exactly the same as the result obtained by backward group preserving scheme [35].
To make a decision on the convergence of the solution, we compute $\alpha_{k}$ using Definition 3 and Theorem 1. For this purpose, let us take $x=y=t=1$; then, 


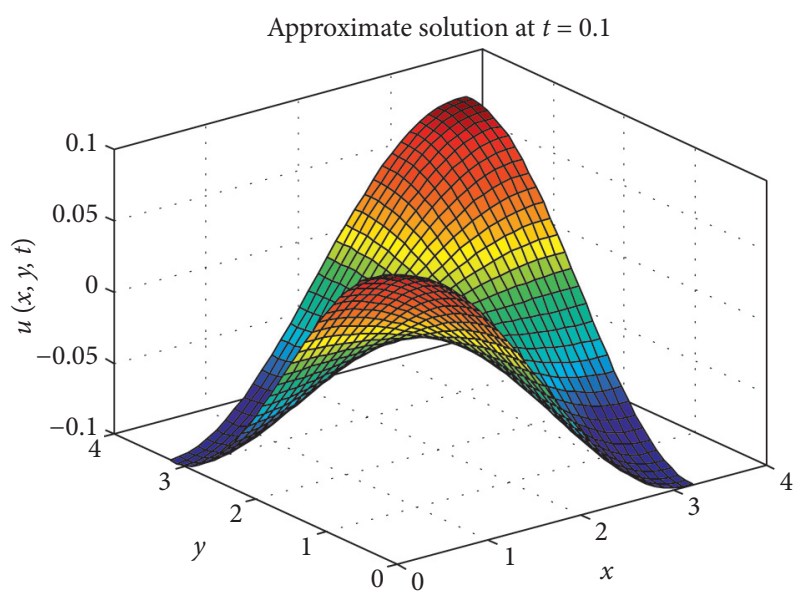

(a)

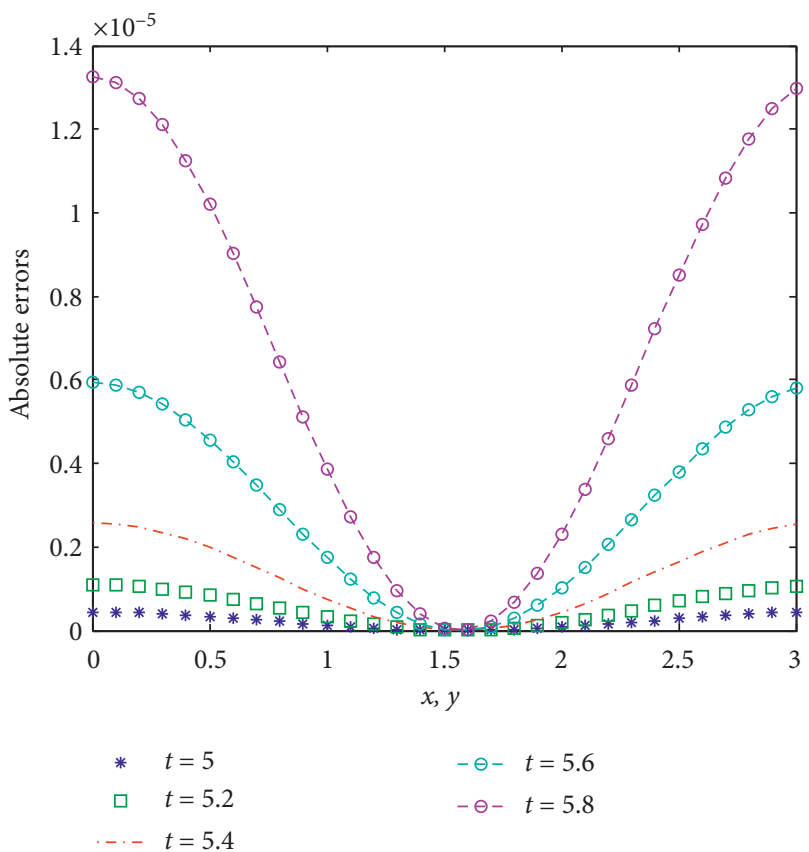

(c)

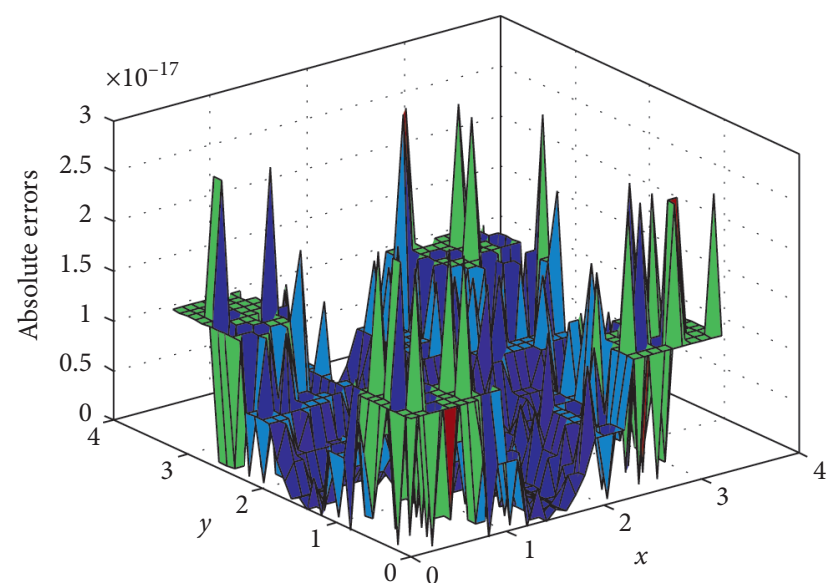

(b)

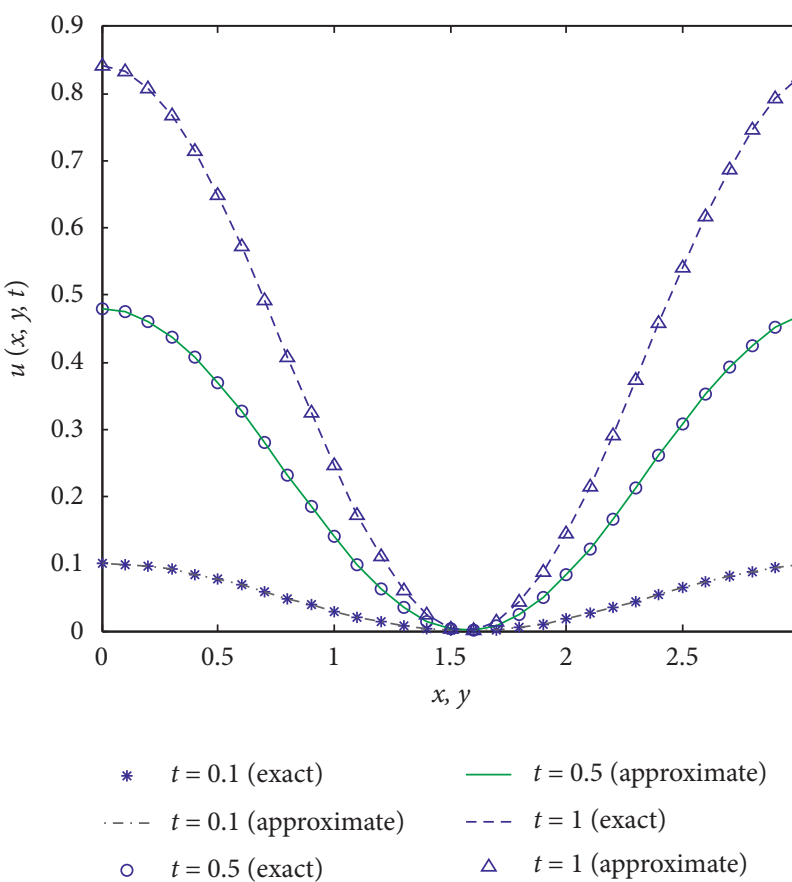

(d)

Figure 2: Continued. 


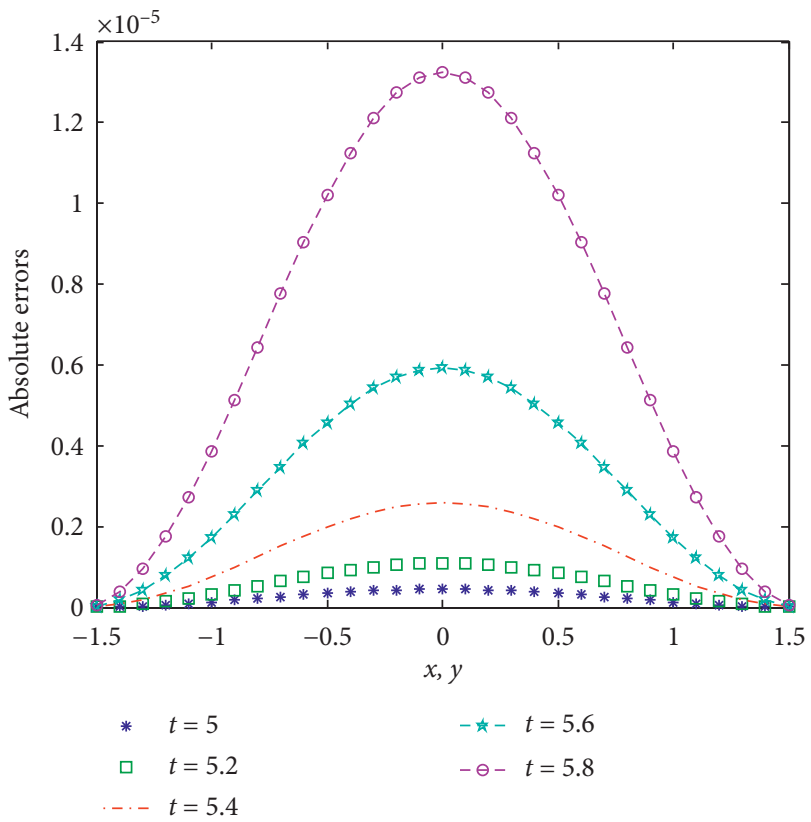

(e)

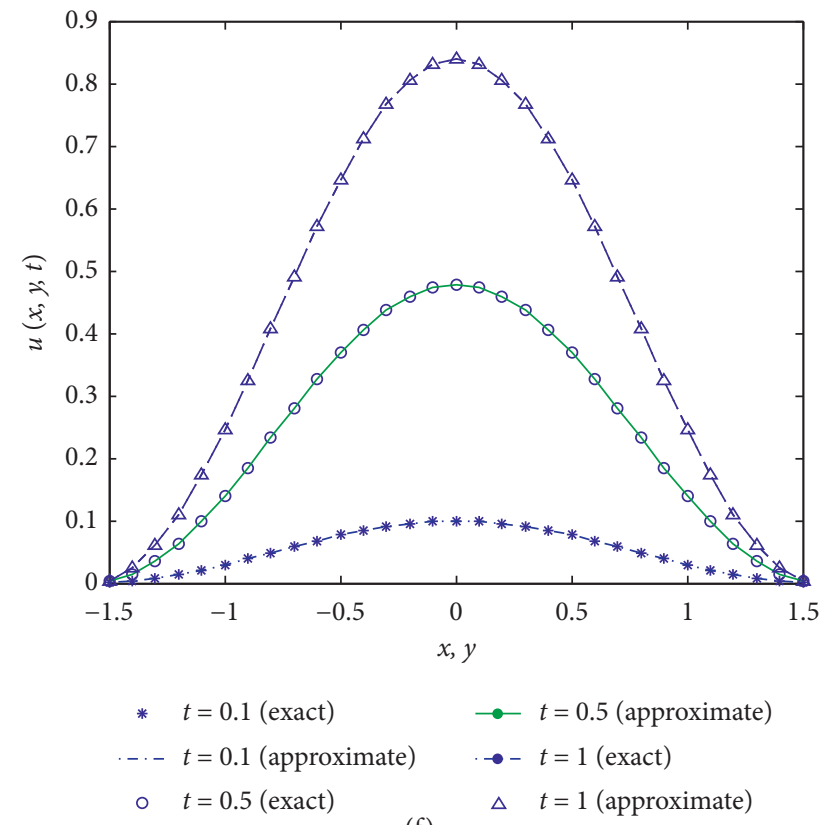

(f)

Figure 2: Plots of the solution behavior of Example 2. (a) Approximate solution at $t=0.1$. (b) Absolute errors at $t=0.1$. (c) Absolute errors for different values of $t$. (d) Comparison of exact and approximate solutions for different values of $t$. (e) Absolute errors for different values of $t$. (f) Comparison of exact and approximate solutions for different values of $t$.

$$
\begin{aligned}
& \alpha_{0}=\frac{\beta_{1}}{\beta_{0}}=0<1, \quad \text { since }\left\|\beta_{0}\right\|=U_{0}(x, y) t^{0}=0, \text { definition } 3, \\
& \alpha_{1}=\frac{\beta_{2}}{\mid \beta_{1}}=\frac{U_{2}(x, y) t^{2}}{U_{1}(x, y) t^{1}}=\frac{0 \times 1^{2}}{0.29192659}=0<1, \\
& \alpha_{2}=\frac{\beta_{3}}{\beta_{2}}=0<1, \quad \text { since }\left\|\beta_{2} \mid\right\|=U_{2}(x, y) t^{2}=0, \text { definition } 3, \\
& \alpha_{3}=\frac{u_{4}}{u_{3}}=\frac{U_{4}(x, y) t^{4}}{U_{3}(x, y) t^{3}}=0<1,
\end{aligned}
$$

and so on

therefore for $x, y, t \geq 0, \alpha_{0}=\alpha_{1}=\alpha_{2}=\alpha_{3}=\ldots=\alpha_{\infty}=0<1$.

So, by Theorem 2, the given NLKGE has a convergent solution.

Figures representing the numerical solution of Example 2 are presented below.

In Figures 2(a) and 2(b), the physical behavior of the approximate solution by RDTM of Example 2 at $t=0.1$ for equal values of $x$ and $y$ in $[0,3.2]$ and the associated absolute errors are shown, respectively. From these figures, it can be seen that the numerical results by RDTM are in excellent agreement with the exact solution of the problem under consideration.

In Figure 2(c), we have plotted absolute errors corresponding to $t=5.0,5.2,5.4,5.6$ and $t=5.8$ for equal values of $x$ and $y$ in $[0,3.2]$ of Example 2. This figure represents the errors of numerical results by RDTM that could appear for the long time range of the given NLKGE.

In Figure 2(d), we have compared the approximate solutions by RDTM corresponding to $t=0.1,0.5$ and $t=1$ with the exact solution, at equal values of $x$ and $y$ in $[0,3.2]$. This figure shows that the numerical results obtained by RDTM are in excellent agreement with the exact results.

Moreover, for equal values of $x$ and $y$ in different intervals, the shape of the parabola changes due to the wave nature of the NLKGEs as shown in Figures 2(e) and 2(f). In general, from Figures 2(a)-2(f), it is evident that the approximate solution rapidly converges to the exact solution. 


\section{Conclusion}

The reduced differential transform method (RDTM) has been applied to find an approximate analytical solution of the two-dimensional nonlinear Klein-Gordon equations. The main advantage of the RDTM is that it provides the user an analytical approximation, in many cases, an exact solution, in a rapidly convergent power series form with elegantly computed terms. Two test examples are presented to show the validity of the method under consideration. The approximate solutions of Example 2 obtained by RDTM are in excellent agreement with the exact solution obtained by the backward group preserving scheme [35]. The result is also depicted in Figure 2.

Consequently, this technique can be applied to many NLPDEs arising from science and engineering fields without requiring linearization, discretization, or perturbation.

\section{Data Availability}

The data used to support the findings of this study areavailable from the corresponding author upon request.

\section{Conflicts of Interest}

The authors declare that there are no conflicts of interest regarding the publication of this paper.

\section{Authors' Contributions}

WGB proposed the main idea of this paper. YOM and AKG supervised her work from the first draft to revision. All authors approved the final manuscript for submission.

\section{Acknowledgments}

The authors thank Jimma University, College of Natural Sciences, and Department of Mathematics for providing the necessary resources during this research.

\section{References}

[1] G. Adomian, Solving Frontier Problems of Physics the Decomposition Method, Kluwer Academy Publishing, London, UK, 1994.

[2] H. Singh, R. Pandey, J. Singh, and M. Tripathi, "A reliable numerical algorithm for fractional advection- dispersion equation arising in contaminant transport through porous media," Physica A: Statistical Mechanics and Its Applications, vol. 527, Article ID 121077, 2019.

[3] H. M. Srivastava, V. P. Dubey, R. Kumar et al., "An efficient computational approach for a fractional-order biological population model with carrying capacity," Chaos, Solitons and Fractals, vol. 138, Article ID 109880, 2020.

[4] P. Veeresha, D. G. Prakasha, D. Kumar, D. Baleanu, and J. Singh, "An efficient computational technique for fractional model of generalized Hirota-Satsuma coupled KdV and coupled mKdV equations," Journal of Computational and Nonlinear Dynamics," Journal of Computational and Nonlinear Dynamics, vol. 15, no. 7, p. 14, Article ID 071003, 2020.

[5] A. Goswami, J. Singh, D. Kumar, and Sushila, "An efficient analytical approach for fractional equal width equations describing hydro-magnetic waves in cold plasma," Physica A: Statistical Mechanics and its , Applications, vol. 524, pp. 563-575, 2019.

[6] F. Ali, Z. Ahmad, M. Arif, I. Khan, and K. S. Nisar, "A time fractional model of generalized Couette flow of couple stress nanofluid with heat and mass transfer: applications in engine oil," IEEE Access, vol. 8, pp. 146944-146966, 2020.

[7] M. Arif, F. Ali, I. Khan, and K. S. Nisar, "A time fractional model with non-singular kernel the generalized Couette flow of couple stress nanofluid," IEEE Access, vol. 8, pp. 7737877395, 2020.

[8] F. Ali, M. Iftikhar, I. Khan et al., "“Time fractional analysis of electro-osmotic flow of Walters's-B fluid with time-dependent temperature and concentration," Alexandria Engineering Journal, vol. 59, no. 1, pp. 25-38, 2019.

[9] B. R. Sontakke, A. Shaikh, and K. S. Nisar, "Approximate solutions of a generalized Hirota-Satsuma coupled KdV and a coupled mKdV systems with time fractional derivatives," Malaysian Journal of Mathematical Sciences, vol. 12, no. 2, pp. 175-196, 2018.

[10] F. Ali, A. Imtiaz, I. Khan, N. A. Sheikh, and D. L. C. Ching, "Hemodynamic flow in a vertical cylinder with heat transfer: two-phase Caputo Fabrizio fractional model," Journal of Magnetics, vol. 23, no. 2, pp. 179-191, 2018.

[11] F. Ali, N. Khan, A. Imtiaz, I. Khan, and N. A. Sheikh, ““'The impact of magnetohydrodynamics and heat transfer on the unsteady flow of Casson fluid in an oscillating cylinder via integral transform: a Caputo-Fabrizio fractional model," Paramana, vol. 93, no. 3, p. 12, 2019.

[12] H. Singh, D. Kumar, J. Singh, and C. S. Singh, "A reliable numerical algorithm for the fractional Klein- Gordon equation," Engineering Transactions, vol. 67, no. 1, pp. 21-34, 2019.

[13] W. Greiner, Relativistic Quantum Mechanics-Wave Equations, Springer-Verlag, Berlin, Germany, 3rd edition, 2000.

[14] B. Bülbül and M. Sezer, "A new approach to numerical solution of nonlinear klein-gordon equation," Mathematical Problems in Engineering, vol. 2013, Article ID 869749, 7 pages, 2013.

[15] X. Li, "Meshless numerical analysis of a class of nonlinear generalized Klein-Gordon equations with a well-posed moving least squares approximation," Applied Mathematical Modelling, vol. 48, pp. 153-182, 2017.

[16] O. Acan and Y. Keskin, "Reduced differential transform method for $(2+1)$ dimensional type of the zakharov-kuznetsov ZKEquations," AIP Conference Proceedings, vol. 1648, Article ID 370015, 2015.

[17] Y. Keskin and G. Oturanc, "Reduced differential transform method for partial differential equations," International Journal of Nonlinear Science, Numerical Simulations, vol. 10, no. 6, pp. 741-749, 2009.

[18] P. K. Gupta, "Approximate analytical solutions of fractional benney-lin equation by reduced differential transform method and the Homotopy perturbation method," Computers \& Mathematics with Applications, vol. 61, no. 9, pp. 28292842, 2011.

[19] N. Taghizadeh, M. Akbari, and M. Shahidi, "Application of reduced differential transform method to the Wu-zhang equation," Australian Journal of Basic and Applied Sciences, vol. 5, no. 5, pp. 565-571, 2011.

[20] R. Arora, M. J. Siddiqui, and V. P. Singh, "Solutions of inviscid burgers' and equal width wave equations by RDTM," International Journal of Applied Physics and Mathematics, vol. 2, no. 3, pp. 212-214, 2012. 
[21] O. T. Kolebaje and E. O. Oyewande, "Numerical solution of the $(2+1)$ dimensional sine-gordon equation by reduced differential transform method," International Journal of Modern Applied Physics, vol. 2, no. 1, pp. 15-26, 2013.

[22] M. O. Al-Amr, "New applications of reduced differential transform method," Alexandria Engineering Journal, vol. 53, no. 1, pp. 243-247, 2014.

[23] H. Jafari, H. K. Jassim, S. P. Moshokoa, V. M. Ariyan, and F. Tchier, "Reduced differential transform method for partial differential equations within local fractional derivative operators," Advances in Mechanical Engineering, vol. 8, no. 4, pp. 1-6, 2016.

[24] B. C. Neog, "Solutions of some system of non-linear PDEs using reduced differential transform method," IOSR Journal of Mathematics, vol. 11, no. 5, pp. 37-44, 2015.

[25] J. Yu, J. Jing, Y. Sun, and S. Wu, " $(n+1)$-Dimensional reduced differential transform method for solving partial differential equations," Applied Mathematics and Computation, vol. 273, pp. 697-705, 2016.

[26] M. Gubes and G. Oturanc, "Approximate solutions of coupled ramani equation by using RDTM with compared DTM and exact solutions," New Trends in Mathematical Sciences, vol. 4, pp. 98-212, 2016.

[27] M. S. Mohamed and K. A. Gepreel, "Reduced differential transform method for nonlinear integral member of kadomtsev-petviashvili hierarchy differential equations," Journal of the Egyptian Mathematical Society, vol. 25, no. 1, pp. 1-7, 2017.

[28] V. K. Srivastava, M. K. Awasthi, and R. K. Chaurasia, "Reduced differential transform method to solve two and three dimensional second order hyperbolic telegraph equations," Journal of King Saud University - Engineering Sciences, vol. 29, no. 2, pp. 166-171, 2017.

[29] M. Gubes and G. Oturanc, "Approximate solutions of coupled Ramani equation by using RDTM with compared DTM and exact solutions," New Trends in Mathematical Science, vol. 4, no. 4 , p. 198,2016

[30] A.-S. J. Al-Saif and A. J. Harfash, "A Comparison between the reduced differential transform method and perturbation-iteration algorithm for solving two-dimensional unsteady incompressible Navier-Stokes equations," Journal of Applied Mathematics and Physics, vol. 06, no. 12, pp. 2518-2543, 2018.

[31] G. Hatıra, "Analytical and approximate solution of two-dimensional convection-diffusion problems," An International Journal of Optimization and Control Theories \& Applications, vol. 10, no. 1, pp. 73-77, 2020.

[32] S. Mohmoud and M. Gubara, "Reduced differential transform method for solving linear and nonlinear goursat problem," Applied Mathematics, vol. 7, no. 10, pp. 1049-1056, 2016.

[33] A. Ziqan, S. Armiti, and I. Suwan, "Solving three-dimensional Volterra integral equation by the reduced differential transform method," International Journal of Applied Mathematical Research, vol. 5, no. 2, pp. 103-106, 2016.

[34] K. Yildirim, "A solution method for solving systems of nonlinear PDEs," World Applied Sciences Journal, vol. 18, no. 11, pp. 1527-1532, 2012.

[35] C.-W. Chang and C.-C. Kuo, "A Lie-group approach for solving backward two-dimensional nonlinear Klein-Gordon equation," Procedia Engineering, vol. 79, pp. 590-598, 2014. 